International Journal of Agriculture, Environment and Bioresearch

Vol. 4, No. 04; 2019

ISSN: $2456-8643$

\title{
ASSESSMENT OF PHENOTYPIC DIVERSITY IN TUNISIAN CARROT (Daucus carota subsp. sativus) LANDRACES
}

\author{
Jihen Benamor ${ }^{1,2^{*}}$, NajlaMezghani $^{2 *}$, David Spooner ${ }^{3}$, KhawlaHamdi ${ }^{1}$, AichaGhezal ${ }^{4}$ and NejiTarchoun1 \\ ${ }^{1}$ Laboratory of vegetable crops, High Agronomic Institute of ChottMariem, Université de Sousse, 1042 Sousse, \\ Tunisia \\ ${ }^{2}$ National Gene Bank of Tunisia, Boulevard Leader Yasser Arafat Z. I Charguia 1, 1080 Tunis, Tunisia \\ ${ }^{3}$ USDA-ARS, Vegetable Crops Research Unit, Department of Horticulture, University of Wisconsin, 1575 Linden \\ Drive, Madison, WI 53706-1590, USA \\ ${ }^{4}$ General Directorate of Agricultural Production, 30 Rue Alain savary, 1002 Tunis, Tunisia
}

http://doi.org/10.35410/IJAEB.2019.4409

Corresponding author: jihen.ben.amor90@gmail.com

* These authors contributed equally to the work.

\begin{abstract}
Knowledge of the morphological diversity of a germplasm collection is fundamental for genebank managers and plant breeders. The main objective of the present work was to characterize 33 landraces of carrot from 13 different regions of Tunisia, based on 34 agromorphological characters related to leaves and roots. The Shannon-Weaver Diversity (H') index was used to study the phenotypic diversity. The estimated H' ranged from 0.19 for core colour compared to cortex colour (RCCCC) to 0.99 for leaf division (LD). Analysis of variance revealed significant differences among landraces for all quantitative characters. Stepwise multivariate analyses were carried out to identify the useful characters that can distinguish among landraces. This study showed that qualitative characters were the best for the delimitation of landraces in this collection. Cluster analysis permitted the subdivision of carrot collection into four distinct groups independent of their geographic distribution. This information will be helpful to curators in the management and improvement of carrot germplasm in Tunisia.
\end{abstract}

Keywords: Carrot, morphological characterization, ANOVA, multivariate analyses.

\section{INTRODUCTION}

The genus Daucus includes about 25 recognized species world-wide. The most widespread and economically important species, Daucus carota L., occurs on almost every continent. It is found in wild or cultivated form throughout the Mediterranean, southwest Asia, Africa, Australia, New Zealand and the Americas (Peterson and Simon, 1986; Vaughan and Geissler,2009). Central Asia is considered the center of origin of cultivated carrot, which represents a large genetic variation (Maksylewiczand Baranski, 2013; Iorizzoet al., 2013). At present, large genetic variation is observed in cultivated carrot (Daucus carota subsp. sativus (Hoffm.) Arcang.) due to the fast spread of carrot ancestors from their center of origin to distant geographical regions, and to the lack of control of random cross pollination among cultivated and wild forms. Edible carrot is one of the main sources of dietary pro-vitamin A carotenoids (Simon, 1990). Variation in the 
carotenoid content and composition largely depends on the cultivar. The intensive selection on carrot led to a morphological diversity observed in leaves and roots with the first domesticates having purple and yellow roots between 11th and 15th centuries in Central Asia, Asia Minor, Western Europe and England (Banga, 1963). Orange carrot roots were domesticated in Europe between 15th and 16th centuries (Banga, 1957; Stolarczykand Janick, 2011). Among Mediterranean regions, Tunisia is considered a center of biodiversity for Daucus and many other crops, with Tunisia having a great diversity of ecosystems and climates (Pottier Alapetite, 1979; Le Floc'het al., 2010). Carrots are widely cultivated throughout Tunisia, with the prevalence in the center (Sidi Bouzid, Kairouan and Sfax), the south (oasis regions), the coast (Nabeul, Monastir and Mahdia), and the north of the country (Kef and Seliana). Annual carrot production is 218.645 tons, representing $5 \%$ of total vegetable production. Carrot is produced on 6700 ha ( $94 \%$ in the winter crop and 6\% in the summer crop; DGPA,2015). Carrot landraces are genetically heterogeneous, resulting from natural processes and farmers' practices. However, the large genetic diversity pooled in landraces is not exploited by carrot improvement programs because of the lack of information on the agro-morphological and molecular characterization of the germplasm. Recently, Mezghaniet al. (2014, 2017) examined the morphological variation within a Daucus collection conserved at the National Gene Bank of Tunisia using fruit, vegetative and flower data. Relative to D. carota, they recognized the following subspecies: capillifolius (Gilli) Arbizu, carota (L.), gummifer (Syme) Hook. fil and sativus with high degrees of diversity. However, the large diversity regarding local germplasm for cultivated carrot needs to be studied based on agro-morphological, biochemical and molecular characterization. Thus, the aims of this study are (1) the morphological characterization of carrot landraces collected from the major growing regions of the country using several vigour descriptors related to leaf and root and (2) the analysis of genetic variation among the accessions using uni and multivariate statistical analysis of the data. This information will guide the curators in the formulation and prioritization of future conservation activities especially in the field of carrot germplasm exploration and enhancement, and guide breeders into choice of germplasm.

\section{MATERIALS AND METHODS}

\subsection{Plant material}

The study material consisted of 33 carrot landraces collected during the harvest period extending between December 2015 and February 2016 from 13 localities belonging to the main cultivation regions in Tunisia. Each accession is represented by 15 plants (roots and leaves parts) collected from the fields and seeds from the farm store. Passport data and an inventory number were assigned for each accession according to the National Gene Bank of Tunisia database and full details are available at the Germplasm Resources Information Network - GRIN (http://www.tngrin.nat.tn/gringlobal/search.aspx). The collection and geographic position are displayed in figure 1 . 


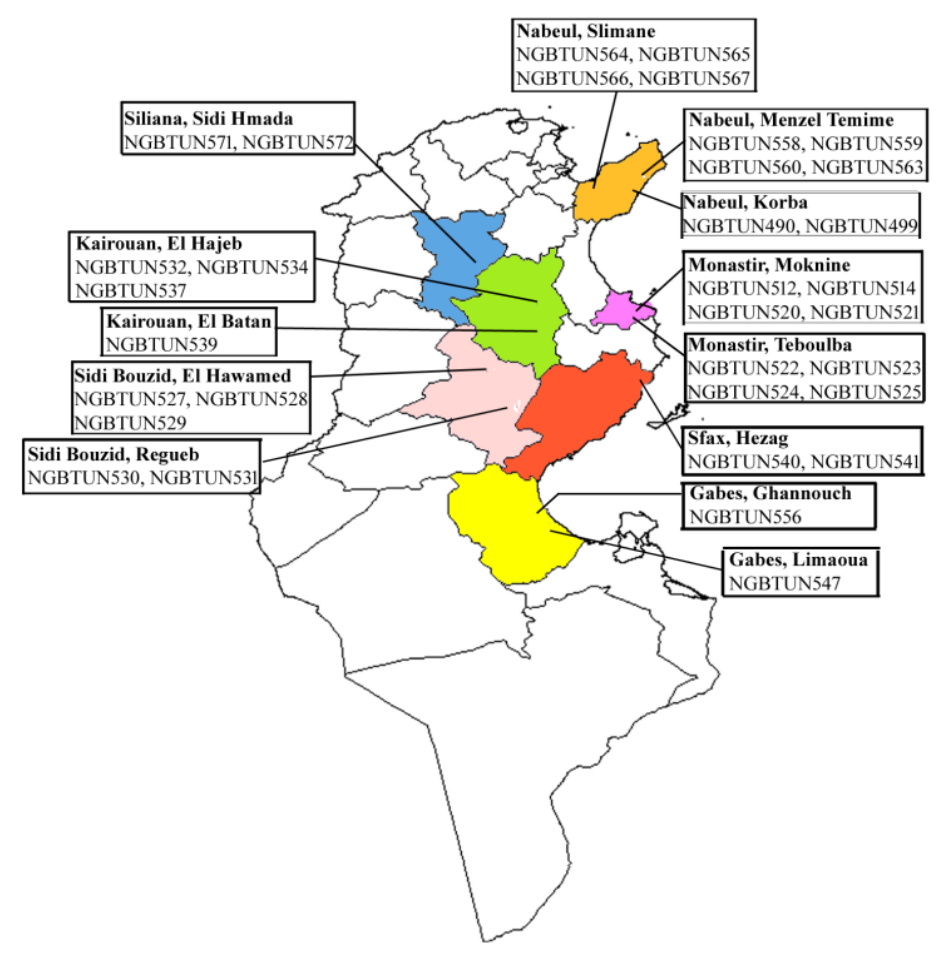

Figure 1: Geographic distribution of carrot collection used in this study. The names of the provinces and locations are in bold. NGBTUN numbers are permanent identification assigned to accessions maintained at the National Gene Bank of Tunisia.

\subsection{Morphological characterization}

Carrot landraces were examined for 15 quantitative and 19 qualitative traits related to roots and leaves (Table 1). The selection of characters was made following the descriptors lists of IPGRI (International Plant Genetic Resources Institute, 1998) and UPOV (International Union for the Protection of New Varieties of Plants, 2007). Quantitative traits (length, width and diameter) were measured with a ruler or caliper, root weight with an electronic balance and root firmness with a penetrometer, whilequalitative traits were evaluated by attributing a code to each character state. 
Table 1: Morphological descriptors, descriptor states, their codes for numerical analysis, frequency distribution and diversity index of carrot landraces in Tunisia

\begin{tabular}{|c|c|c|c|c|c|c|c|}
\hline Trait/descriptor & Source & Descriptor acronym & Type & Descriptor state $^{a}$ & Class & Frequency (\%) & $\begin{array}{l}\text { Diversity } \\
\text { Index (H') }\end{array}$ \\
\hline \multicolumn{8}{|l|}{ Leaf } \\
\hline Crown width & UPOV & $\mathrm{CW}$ & QL & $\begin{array}{l}\text { Narrow } \\
\text { Medium } \\
\text { Broad }\end{array}$ & $\begin{array}{l}3 \\
5 \\
7\end{array}$ & $\begin{array}{l}66.4 \\
25.1 \\
8.5\end{array}$ & 0.75 \\
\hline Leafnumber & IPGRI & LN & QN & $\begin{array}{l}\text { Low }(\leq 5.2) \\
\text { Medium }(5.2-14.29) \\
\text { High }(\geq 14.29)\end{array}$ & $\begin{array}{l}1 \\
2 \\
3\end{array}$ & $\begin{array}{l}6.1 \\
80.6 \\
13.3\end{array}$ & 0.55 \\
\hline Leaflength $(\mathrm{cm})$ & IPGRI & LL & QN & $\begin{array}{l}\text { Short }(\leq 42.4) \\
\text { Intermediate (42.4-73.8) } \\
\text { Elongated }(\geq 73.8)\end{array}$ & $\begin{array}{l}1 \\
2 \\
3\end{array}$ & $\begin{array}{r}13.9 \\
66.7 \\
19.4\end{array}$ & 0.78 \\
\hline Leafwidth $(\mathrm{cm})$ & IPGRI & LW & QN & $\begin{array}{l}\text { Narrow }(\leq 16.5) \\
\text { Intermediate }(16.5-36.1) \\
\text { Wide }(\geq 36.1)\end{array}$ & $\begin{array}{l}1 \\
2 \\
3\end{array}$ & $\begin{array}{r}13.3 \\
73.4 \\
13.3\end{array}$ & 0.69 \\
\hline Leaf division & UPOV & LD & QL & $\begin{array}{l}\text { Fine } \\
\text { Medium } \\
\text { Coarse }\end{array}$ & $\begin{array}{l}3 \\
5 \\
5 \\
7\end{array}$ & $\begin{array}{l}30.6 \\
39.7 \\
29.7\end{array}$ & 0.99 \\
\hline Intensity of green colour & UPOV & LIGC & QL & $\begin{array}{l}\text { Light } \\
\text { Medium } \\
\text { Dark }\end{array}$ & $\begin{array}{l}3 \\
5 \\
7\end{array}$ & $\begin{array}{l}33.0 \\
51.2 \\
15.8\end{array}$ & 0.91 \\
\hline Leafhairiness & IPGRI & LH & QL & $\begin{array}{l}\text { Sparse } \\
\text { Intermediate } \\
\text { Dense }\end{array}$ & $\begin{array}{l}3 \\
5 \\
7\end{array}$ & $\begin{array}{l}73.0 \\
13.7 \\
13.3\end{array}$ & 0.70 \\
\hline Leafletsnumber & IPGRI & LIN & QN & $\begin{array}{l}\text { Low }(\leq 21.3) \\
\text { Medium (21.3-28.7) } \\
\text { High }(\geq 28.7)\end{array}$ & $\begin{array}{l}1 \\
2 \\
3\end{array}$ & $\begin{array}{l}9.4 \\
79.4 \\
11.2\end{array}$ & 0.59 \\
\hline Length of primary basal leaflet $(\mathrm{cm})$ & IPGRI & LPBL & QN & $\begin{array}{l}\text { Short }(\leq 10.1) \\
\text { Intermediate (10.1-22.6) } \\
\text { Elongated }(\geq 22.6)\end{array}$ & $\begin{array}{l}1 \\
2 \\
3\end{array}$ & $\begin{array}{l}15.1 \\
68.8 \\
16.1\end{array}$ & 0.76 \\
\hline Number of segments of primary basal leaflet & IPGRI & NSPBL & QN & $\begin{array}{l}\text { Low }(\leq 15.0) \\
\text { Medium }(15.0-19.7) \\
\text { High }(\geq 19.7)\end{array}$ & $\begin{array}{l}1 \\
2 \\
3\end{array}$ & $\begin{array}{l}17.9 \\
57.3 \\
24.8\end{array}$ & 0.88 \\
\hline Foliage coverage & IPGRI & FC & QL & $\begin{array}{l}\text { Sparse } \\
\text { Dense }\end{array}$ & $\begin{array}{l}3 \\
7\end{array}$ & $\begin{array}{l}42.4 \\
57.6\end{array}$ & 0.98 \\
\hline Petioleanthocyanincolouration & IPGRI & PCP & QL & $\begin{array}{l}\text { Uncoloured } \\
\text { Slightly coloured } \\
\text { Intermediate } \\
\text { Strongly coloured }\end{array}$ & $\begin{array}{l}1 \\
3 \\
5 \\
7\end{array}$ & $\begin{array}{l}72.8 \\
19.7 \\
6.9 \\
0.6\end{array}$ & 0.55 \\
\hline Petiolethickness (mm) & IPGRI & PT & QN & $\begin{array}{l}\text { Narrow }(\leq 3.6) \\
\text { Intermediate }(3.6-7.4) \\
\text { Wide }(\geq 7.4)\end{array}$ & $\begin{array}{l}1 \\
2 \\
3\end{array}$ & $\begin{array}{l}12.4 \\
70.9 \\
16.7\end{array}$ & 0.72 \\
\hline Root & & & & & & & \\
\hline Root length $(\mathrm{cm})$ & IPGRI & RL & QN & Short $(\leq 21.4)$ & 1 & 16.0 & 0.77 \\
\hline
\end{tabular}


International Journal of Agriculture, Environment and Bioresearch

Vol. 4, No. 04; 2019

ISSN: $2456-8643$

\begin{tabular}{|c|c|c|c|c|c|c|c|}
\hline & & & & $\begin{array}{l}\text { Intermediate (21.4-31.8) } \\
\text { Elongated }(\geq 31.8)\end{array}$ & 3 & $\begin{array}{l}68.2 \\
15.8\end{array}$ & \\
\hline Root diameter at the shoulder $(\mathrm{mm})$ & IPGRI & RDS & QN & $\begin{array}{l}\text { Narrow }(\leq 32.4) \\
\text { Intermediate }(32.4-53.0) \\
\text { Wide }(\geq 53.0)\end{array}$ & 1 & $\begin{array}{r}12.1 \\
70.0 \\
17.9\end{array}$ & 0.74 \\
\hline Root diameter at the medium (mm) & IPGRI & RDMd & QN & $\begin{array}{l}\text { Narrow }(\leq 27.9) \\
\text { Intermediate }(27.9-45.2) \\
\text { Wide }(\geq 45.2)\end{array}$ & 1 & $\begin{array}{l}12.7 \\
74.3 \\
13.0\end{array}$ & 0.68 \\
\hline Root diameter at the tip $(\mathrm{mm})$ & IPGRI & RDTi & QN & $\begin{array}{l}\text { Narrow }(\leq 14.0) \\
\text { Intermediate }(14.0-31.7) \\
\text { Wide }(\geq 31.7)\end{array}$ & 1 & $\begin{array}{l}15.8 \\
68.2 \\
16.0\end{array}$ & 0.77 \\
\hline Root weight (Kg) & IPGRI & RW & QN & $\begin{array}{l}\text { Light }(\leq 0.09) \\
\text { Intermediate }(0.09-0.40) \\
\text { Heavy }(\geq 0.40)\end{array}$ & 1 & $\begin{array}{l}5.5 \\
84.2 \\
10.3\end{array}$ & 0.48 \\
\hline Root axis & IPGRI & RA & QL & $\begin{array}{l}\text { Not straight } \\
\text { Straight }\end{array}$ & 1 & $\begin{array}{l}30.3 \\
69.7\end{array}$ & 0.88 \\
\hline Root shape in longitudinal section & UPOV & RSLS & QL & $\begin{array}{l}\text { Obovate } \\
\text { Medium obtriangular } \\
\text { Narrow obtriangular } \\
\text { Narrow obtriangular to narrow } \\
\text { oblong } \\
\text { Narrow oblong }\end{array}$ & 2 & \begin{tabular}{|l}
0.6 \\
9.4 \\
58.4 \\
17.9 \\
\\
13.7
\end{tabular} & 0.71 \\
\hline Root shoulder shape & UPOV & RSS & QL & $\begin{array}{l}\text { Flat } \\
\text { Flat to rounded } \\
\text { Rounded } \\
\text { Rounded to conical } \\
\text { Conical }\end{array}$ & 1 & $\begin{array}{l}4.3 \\
76.0 \\
16.4 \\
0.3 \\
3.0\end{array}$ & 0.47 \\
\hline Root tip shape & UPOV & RTS & QL & $\begin{array}{l}\text { Blunt } \\
\text { Slightly pointed } \\
\text { Strongly pointed }\end{array}$ & 3 & $\begin{array}{l}14.9 \\
30.3 \\
54.8\end{array}$ & 0.88 \\
\hline Root external colour & UPOV & REC & QL & $\begin{array}{l}\text { Yellow } \\
\text { Orange } \\
\text { Pinkish red } \\
\text { Purple }\end{array}$ & 2 & $\begin{array}{l}22.1 \\
57.9 \\
17.2 \\
2.8\end{array}$ & 0.75 \\
\hline Anthocyanin colouration of shoulder skin & UPOV & RACSS & QL & $\begin{array}{l}\text { Absent } \\
\text { Present }\end{array}$ & 9 & $\begin{array}{l}37.6 \\
62.4\end{array}$ & 0.95 \\
\hline Extent of green colour of shoulder skin & UPOV & REGCSS & QL & $\begin{array}{l}\text { Absent or very small } \\
\text { Small } \\
\text { Medium } \\
\text { Large }\end{array}$ & 1 & $\begin{array}{l}56.6 \\
1.2 \\
2.8 \\
39.4\end{array}$ & 0.6 \\
\hline Surface ridging & UPOV & RSR & QL & $\begin{array}{l}\text { Absent or very weak } \\
\text { Weak } \\
\text { Medium }\end{array}$ & 5 & $\begin{array}{l}69.1 \\
27.6 \\
3.3\end{array}$ & 0.65 \\
\hline Core diameter $(\mathrm{mm})$ & IPGRI & RCD & QN & $\begin{array}{l}\text { Small }(\leq 14.1) \\
\text { Intermediate }(14.1-25.2) \\
\text { Large }(\geq 25.2)\end{array}$ & 3 & $\begin{array}{l}14.6 \\
71.5 \\
13.9\end{array}$ & 0.72 \\
\hline Cortex diameter $(\mathrm{mm})$ & UPOV & RCortD & QN & Narrow $(\leq 5.2)$ & 1 & 11.5 & 0.59 \\
\hline
\end{tabular}


ISSN: 2456-8643

\begin{tabular}{|c|c|c|c|c|c|c|c|}
\hline & & & & $\begin{array}{l}\text { Intermediate (5.2-10.5) } \\
\text { Wide }(\geq 10.5)\end{array}$ & $\begin{array}{l}2 \\
3\end{array}$ & $\begin{array}{l}79.1 \\
9.4\end{array}$ & \\
\hline Core colour & UPOV & RCC & $\mathrm{QL}$ & $\begin{array}{l}\text { White } \\
\text { Yellow } \\
\text { Orange } \\
\text { Pinkish red }\end{array}$ & $\begin{array}{l}1 \\
2 \\
3 \\
4\end{array}$ & $\begin{array}{l}2.5 \\
73.0 \\
22.4 \\
2.1\end{array}$ & 0.53 \\
\hline Core colour compared to root cortex colour & UPOV & RCCCC & QL & $\begin{array}{l}\text { Lighter } \\
\text { Same } \\
\text { Darker }\end{array}$ & $\begin{array}{l}1 \\
2 \\
3\end{array}$ & $\begin{array}{l}94.9 \\
4.8 \\
0.3\end{array}$ & 0.19 \\
\hline Root branching & IPGRI & RB & QL & $\begin{array}{l}\text { Absent } \\
\text { Sparse } \\
\text { Intermediate }\end{array}$ & $\begin{array}{l}0 \\
3 \\
5\end{array}$ & $\begin{array}{l}85.1 \\
13.0 \\
1.9\end{array}$ & 0.43 \\
\hline Flesh colour distribution in transverse section & IPGRI & RFCDTS & $\mathrm{QL}$ & $\begin{array}{l}\text { Colour in two distinct outer and } \\
\text { inner cores } \\
\text { Colour radially distributed in } \\
\text { stellate pattern } \\
\text { Colour radially distributed from } \\
\text { inner core }\end{array}$ & 4 & $\begin{array}{l}79.1 \\
20.6 \\
0.3\end{array}$ & 0.48 \\
\hline Root firmness & UPOV & $\mathrm{RF}$ & QN & $\begin{array}{l}\text { Low }(\leq 3.3) \\
\text { Intermediate }(3.3-5.9) \\
\operatorname{High}(\geq 5.9)\end{array}$ & $\begin{array}{l}1 \\
2 \\
3\end{array}$ & $\begin{array}{r}16.4 \\
67.9 \\
15.7\end{array}$ & 0.77 \\
\hline Protrusion above soil & UPOV & RPAS & QL & $\begin{array}{l}\text { Small } \\
\text { Medium } \\
\text { Large }\end{array}$ & $\begin{array}{l}3 \\
5 \\
7\end{array}$ & $\begin{array}{l}6.0 \\
36.4 \\
57.6\end{array}$ & 0.77 \\
\hline
\end{tabular}

aQuantitative characters were converted to phenotypic classes with the class boundaries as described by Jaradatet al. (2004); QN: quantitative, QL: qualitative.

\subsection{Statistical analyses}

Data analyses were performed using statistical procedures in SAS 9.1 software (SAS 1990). Simple statistics such as means and coefficient of variation were used on quantitative parameters to compare the variation among the landraces. A variance analysis (ANOVA) was performed and then the averages were compared by Duncan's multiple range test. A Pearson correlation analysis was then carried out to estimate the relationship between the studied variables. The following multivariate analyses were performed to evaluate the contribution of each quantitative and qualitative character to the total variation: Principal component analysis (PCA), factorial correspondence analysis (FCA) and hierarchical cluster analysis (HCA) were conducted on quantitative, qualitative and mixed data respectively. For calculating the diversity parameters, the overall entry mean value and the standard deviation were used to convert quantitative characters into qualitative ones (Jaradatet al., 2004) and frequencies were obtained from class intervals. The diversity was measured for each morphological character by using the standardized Shannon-Weaver (Shannon and Weaver, 1949; as referred by Al Khanjariet al., 2008).Diversity Index, designed as $\mathrm{H}^{\prime}$ has the formula: $\mathrm{H}^{\prime}=-\sum$ pi $(\log 2$ pi) / $\log 2$ n, where pi $=$ frequency proportion of each descriptor state and $\mathrm{n}=$ number of states for each descriptor. The diversity index was coded as high $\left(H^{\prime} \geq 0.60\right)$, intermediate $\left(0.40 \leq H^{\prime}<0.60\right)$ or low $\left(0.10 \leq H^{\prime}<0.40\right)$ as described by Etichaet al. (2005).

\section{RESULTS}

\subsection{Diversity analysis}

Large natural variation was found among landraces for the majority of traits (Table 1). The diversity index (H') ranged from 0.19 for core colour compared to cortex colour (RCCCC) to 0.99 for leaf division (LD) with an overall mean of 0.69 . The majority of traits (13 qualitative and 11 quantitative) showed a high level of polymorphism $\left(H^{\prime} \geq 0.6\right)$. Intermediate variation $\left(0.4 \leq H^{\prime}<0.6\right)$ was observed in 9 characters. Core colour compared to cortex colour $(\mathrm{RCCCC})$ was the only character exhibiting low level of variation $\left(\mathrm{H}^{\prime}=0.19\right)$. High variation indicates equitable distribution of the different states while low variation indicates the dominance of one character state over the others as shown by frequency distribution (Mengistu et al., 2015). Research performed by Mezghaniet al. (2017), on morphological variation of 45 Daucus carota L. accessions in Tunisia showed high overall mean diversity indexes in quantitative $\left(H^{\prime}=0.77\right)$ and qualitative $\left(H^{\prime}=0.75\right)$ characters confirming that Tunisia is a principal major center of diversification for carrot and wild relatives in the Mediterranean region.

\subsection{Phenetic analysis}


ISSN: $2456-8643$

\subsubsection{Quantitative characters}

Analysis of variance for 15 quantitative data showed high significant differences $(\mathrm{p}<0.0001)$ for all recorded traits among the landraces (Table 2). The coefficient of variation ranged from 13.58\% (lowest) to $46.82 \%$ (highest) for number of leaflets (LIN) and root weight $(\mathrm{RW})$ respectively. The high coefficients of variation $(\geq 20 \%)$ observed for 7 characters signify a high degree of variability for effective selection of landraces. An important variability was also detected in morphological characters related to roots and leaves of yellow carrot accessions in Iran (Kasiriet al., 2013; Mehrabiet al., 2014). The degree of genetic variability within crop species is a function of the method of their domestication, the breeding system and the method by which it is maintained (Hamrick et al., 1979).

Table 2:Means comparison for quantitative traits in 33 Tunisian carrot landraces. Means in the same column followed by the same letter are not significant different at $\mathbf{P}<\mathbf{0 . 0 5}$ according to Duncan's multiple range test.

\begin{tabular}{|c|c|c|c|c|c|c|c|c|c|c|c|c|c|c|c|}
\hline Accession & $L N$ & $L L$ & $L W$ & $L I N$ & $L P B L$ & $N S P B L$ & $P T$ & $R L$ & $R D S$ & $R D M d$ & $R D T i$ & $R W$ & $R C D$ & RCortD & $R F$ \\
\hline NGBTUN490 & $8.60 \mathrm{fgh}$ & $45.24 \mathrm{klm}$ & $10.30 \mathrm{q}$ & $24.00 \mathrm{fgh}$ & $10.83 \mathrm{mn}$ & 15.80fgh & $3.83 \mathrm{jk}$ & $24.00 \mathrm{ijk}$ & $33.77 \mathrm{mno}$ & $29.651 \mathrm{mn}$ & $20.47 \mathrm{jkl}$ & $0.09 \mathrm{j}$ & $16.921 \mathrm{mn}$ & $6.78 \mathrm{klm}$ & $6.04 a b c$ \\
\hline NGBTUN499 & $8.60 \mathrm{fgh}$ & $48.60 \mathrm{klm}$ & 16.10nop & $22.40 \mathrm{gh}$ & 15.50hij & 17.00fgh & 5.23fgh & $23.50 \mathrm{ijk}$ & $40.39 \mathrm{klm}$ & $33.40 \mathrm{jkl}$ & $21.33 \mathrm{jkl}$ & $0.11 \mathrm{ij}$ & $19.78 \mathrm{ijk}$ & $6.28 \mathrm{klm}$ & $6.37 \mathrm{a}$ \\
\hline NGBTUN512 & $6.90 \mathrm{hi}$ & 79.18ab & 27.73hij & $25.20 \mathrm{fgh}$ & $22.26 \mathrm{bcd}$ & 16.10fgh & $7.02 \mathrm{~d}$ & 28.92def & $35.80 \mathrm{mno}$ & $31.391 \mathrm{mn}$ & $15.931 \mathrm{mn}$ & $0.16 \mathrm{hij}$ & $17.98 \mathrm{klm}$ & $6.56 \mathrm{klm}$ & 5.14def \\
\hline NGBTUN514 & $11.20 \mathrm{edf}$ & $73.03 \mathrm{bc}$ & $39.70 \mathrm{bc}$ & $26.80 \mathrm{cde}$ & $25.39 \mathrm{ab}$ & $17.80 \mathrm{def}$ & $7.31 \mathrm{~d}$ & 26.42hij & $39.511 \mathrm{mn}$ & $39.52 \mathrm{efg}$ & $18.42 \mathrm{klm}$ & $0.28 \mathrm{cde}$ & $23.53 \mathrm{cde}$ & 7.49ijk & $6.14 a b$ \\
\hline NGBTUN520 & 7.80fgh & $79.41 \mathrm{ab}$ & $36.05 \mathrm{~cd}$ & $25.70 \mathrm{fgh}$ & $20.86 \mathrm{~cd}$ & 15.50fgh & $7.61 \mathrm{~cd}$ & 29.03def & $34.08 \mathrm{mno}$ & $30.271 \mathrm{mn}$ & $13.42 \mathrm{mn}$ & $0.13 \mathrm{hij}$ & $19.01 \mathrm{klm}$ & $4.82 \mathrm{~m}$ & $5.27 \mathrm{def}$ \\
\hline NGBTUN521 & 9.50fgh & $70.50 \mathrm{~cd}$ & $32.95 \mathrm{efg}$ & $26.00 \mathrm{efg}$ & $22.87 b c$ & $15.40 \mathrm{gh}$ & $7.61 \mathrm{~cd}$ & 27.14ghi & $38.761 \mathrm{mn}$ & $36.72 \mathrm{ghi}$ & $19.14 \mathrm{klm}$ & $0.21 \mathrm{hij}$ & $20.20 \mathrm{ijk}$ & $6.47 \mathrm{klm}$ & $5.59 \mathrm{bcd}$ \\
\hline NGBTUN522 & $7.00 \mathrm{hi}$ & $83.23 \mathrm{a}$ & $26.50 \mathrm{ijk}$ & $27.40 \mathrm{bcd}$ & $26.69 \mathrm{a}$ & $18.70 \mathrm{abc}$ & $7.51 \mathrm{~d}$ & $29.32 \mathrm{cde}$ & 42.64ghi & 36.08hij & $16.391 \mathrm{mn}$ & $0.24 \mathrm{fgh}$ & $22.19 \mathrm{efg}$ & $6.97 \mathrm{klm}$ & 4.62efg \\
\hline NGBTUN523 & 10.00fgh & $71.91 \mathrm{~cd}$ & $33.20 \mathrm{efg}$ & $28.50 \mathrm{ab}$ & $25.28 \mathrm{ab}$ & $18.20 \mathrm{bcd}$ & $8.50 \mathrm{bc}$ & 25.00hij & 42.02hij & $35.45 \mathrm{ijk}$ & $18.67 \mathrm{klm}$ & $0.26 \mathrm{efg}$ & $21.99 \mathrm{efg}$ & 7.49ijk & $3.25 \mathrm{ij}$ \\
\hline NGBTUN524 & 7.90fgh & $85.44 \mathrm{a}$ & $42.40 \mathrm{ab}$ & $27.60 \mathrm{abc}$ & $27.71 \mathrm{a}$ & $17.60 \mathrm{efg}$ & $9.30 \mathrm{ab}$ & 26.90hij & $40.50 \mathrm{klm}$ & $34.77 \mathrm{jkl}$ & $20.61 \mathrm{jkl}$ & $0.26 \mathrm{def}$ & $22.49 \mathrm{def}$ & $6.81 \mathrm{klm}$ & 4.39fgh \\
\hline NGBTUN525 & 9.90fgh & $82.69 a$ & $46.37 \mathrm{a}$ & $29.80 \mathrm{a}$ & 25.41ab & $17.50 \mathrm{fgh}$ & $9.46 \mathrm{a}$ & 31.10ab & 42.03hij & $36.63 \mathrm{ghi}$ & $21.39 \mathrm{jkl}$ & $0.30 \mathrm{bc}$ & $21.66 \mathrm{fgh}$ & $7.34 \mathrm{jkl}$ & $5.05 \mathrm{def}$ \\
\hline NGBTUN527 & $17.40 \mathrm{a}$ & 52.10hij & 33.80def & $26.60 \mathrm{def}$ & $16.02 \mathrm{fgh}$ & $18.20 \mathrm{bcd}$ & 4.48hij & 24.40hij & 44.66efg & $34.40 \mathrm{jkl}$ & $26.73 \mathrm{efg}$ & $0.20 \mathrm{hij}$ & $18.82 \mathrm{klm}$ & 7.70ijk & $5.49 \mathrm{bcd}$ \\
\hline NGBTUN528 & 15.10ab & $40.75 \mathrm{mn}$ & 23.90jkl & $26.20 \mathrm{def}$ & $11.251 \mathrm{mn}$ & 18.60abc & 3.89jk & 27.70ghi & 43.37fgh & 36.01hij & 24.63ghi & $0.20 \mathrm{hij}$ & $17.341 \mathrm{mn}$ & 8.37hij & 4.64efg \\
\hline NGBTUN529 & $16.00 \mathrm{ab}$ & $35.05 n$ & 28.30hij & $24.60 \mathrm{fgh}$ & $10.79 \mathrm{mn}$ & 17.00fgh & $3.93 \mathrm{jk}$ & 25.90hij & $42.75 \mathrm{ghi}$ & 37.21 ghi & $27.37 \mathrm{efg}$ & 0.22 ghi & 21.04hij & 8.79ghi & $5.36 \mathrm{cde}$ \\
\hline NGBTUN530 & 11.10efg & $55.19 \mathrm{ghi}$ & 28.40ghi & $24.20 \mathrm{fgh}$ & $17.32 \mathrm{ef}$ & $17.60 \mathrm{efg}$ & 4.54hij & $28.55 \mathrm{efg}$ & $55.39 \mathrm{bc}$ & $42.94 \mathrm{~cd}$ & $30.85 \mathrm{bcd}$ & $0.41 b$ & $25.51 \mathrm{abc}$ & $9.78 \mathrm{def}$ & $5.57 \mathrm{bcd}$ \\
\hline NGBTUN531 & $11.60 \mathrm{cde}$ & $43.351 \mathrm{mn}$ & 27.50hij & $25.00 \mathrm{fgh}$ & $14.59 \mathrm{klm}$ & $18.40 \mathrm{bcd}$ & $4.34 \mathrm{ijk}$ & $29.05 \mathrm{def}$ & 49.36de & $41.89 \mathrm{cde}$ & $29.72 \mathrm{cde}$ & $0.29 \mathrm{~cd}$ & 21.37ghi & $8.92 \mathrm{fgh}$ & $5.12 \mathrm{def}$ \\
\hline NGBTUN532 & 9.50fgh & 54.49ghi & $25.20 \mathrm{jkl}$ & $22.40 \mathrm{gh}$ & $13.571 \mathrm{mn}$ & 17.20fgh & 4.73hij & 26.20hij & $45.24 \mathrm{efg}$ & $39.41 \mathrm{efg}$ & $18.80 \mathrm{klm}$ & $0.20 \mathrm{hij}$ & $21.23 \mathrm{ghi}$ & $9.58 \mathrm{efg}$ & 4.70efg \\
\hline NGBTUN534 & $14.70 \mathrm{abc}$ & $51.25 \mathrm{ijk}$ & $24.70 \mathrm{jkl}$ & $26.80 \mathrm{cde}$ & $15.09 \mathrm{ijk}$ & $18.20 \mathrm{bcd}$ & $5.06 \mathrm{ghi}$ & $23.25 \mathrm{ijk}$ & $51.14 \mathrm{~cd}$ & $38.41 \mathrm{fgh}$ & $18.62 \mathrm{klm}$ & $0.26 \mathrm{efg}$ & $19.42 \mathrm{jkl}$ & $10.21 \mathrm{cde}$ & 4.99def \\
\hline NGBTUN537 & 15.20ab & $41.261 \mathrm{mn}$ & 26.10jkl & $23.80 \mathrm{fgh}$ & $12.811 \mathrm{mn}$ & $17.80 \mathrm{def}$ & 4.72hji & $29.57 \mathrm{bcd}$ & 42.90ghi & 37.61ghi & $12.98 \mathrm{mn}$ & $0.28 \mathrm{cde}$ & $27.92 \mathrm{a}$ & $11.74 \mathrm{bc}$ & 4.92def \\
\hline NGBTUN539 & $13.70 \mathrm{bcd}$ & $40.60 \mathrm{mn}$ & $25.50 \mathrm{jkl}$ & $23.80 \mathrm{fgh}$ & $14.92 \mathrm{jkl}$ & $16.40 \mathrm{fgh}$ & 5.17ghi & $30.43 \mathrm{abc}$ & $36.241 \mathrm{mn}$ & $26.37 n$ & $10.65 n$ & $0.14 \mathrm{hij}$ & $15.811 \mathrm{mn}$ & $5.82 \mathrm{klm}$ & 3.68hij \\
\hline NGBTUN540 & $13.80 \mathrm{bcd}$ & $60.65 \mathrm{egf}$ & $34.80 \mathrm{cde}$ & $26.00 \mathrm{efg}$ & $19.30 \mathrm{de}$ & $20.60 \mathrm{a}$ & $5.97 \mathrm{e}$ & 26.94hij & $71.32 \mathrm{a}$ & $60.91 \mathrm{a}$ & $39.02 \mathrm{a}$ & $0.62 \mathrm{a}$ & $26.14 \mathrm{ab}$ & $15.74 \mathrm{a}$ & $5.59 \mathrm{bcd}$ \\
\hline NGBTUN541 & 10.00 fgh & $41.201 \mathrm{mn}$ & $20.30 \mathrm{mno}$ & $22.00 \mathrm{~h}$ & $11.601 \mathrm{mn}$ & $15.20 \mathrm{~h}$ & 4.87hij & 24.75hij & $37.051 \mathrm{mn}$ & $30.131 \mathrm{mn}$ & $16.981 \mathrm{mn}$ & $0.12 \mathrm{hij}$ & $14.521 \mathrm{mn}$ & $5.83 \mathrm{klm}$ & $4.65 \mathrm{efg}$ \\
\hline NGBTUN547 & 6.90hi & 54.60ghi & $22.001 \mathrm{mn}$ & $24.00 \mathrm{fgh}$ & $12.451 \mathrm{mn}$ & $18.00 \mathrm{cde}$ & $4.31 \mathrm{ijk}$ & 17.721 & $59.26 \mathrm{~b}$ & $51.32 \mathrm{~b}$ & $34.25 \mathrm{ab}$ & $0.25 \mathrm{efg}$ & $24.27 \mathrm{bcd}$ & $12.47 \mathrm{~b}$ & 6.03abc \\
\hline
\end{tabular}


ISSN: 2456-8643

\begin{tabular}{|c|c|c|c|c|c|c|c|c|c|c|c|c|c|c|c|}
\hline NGBTUN556 & $6.40 \mathrm{hi}$ & 52.35hij & 14.70opq & 23.80fgh & $13.301 \mathrm{mn}$ & $15.40 \mathrm{gh}$ & 4.77hij & $19.96 \mathrm{kl}$ & $55.74 \mathrm{bc}$ & $46.54 \mathrm{bc}$ & $32.33 b c$ & $0.23 \mathrm{ghi}$ & $21.86 \mathrm{efg}$ & $10.73 \mathrm{bcd}$ & $5.10 \mathrm{def}$ \\
\hline NGBTUN558 & $6.90 \mathrm{hi}$ & $55.76 \mathrm{ghi}$ & $24.60 \mathrm{jkl}$ & 24.60fgh & $14.03 \mathrm{klm}$ & $17.40 \mathrm{fgh}$ & $5.26 \mathrm{fgh}$ & 24.87hij & $36.621 \mathrm{mn}$ & 35.84hij & $22.08 \mathrm{jkl}$ & 0.16hij & $16.681 \mathrm{mn}$ & $6.82 \mathrm{klm}$ & $2.97 \mathrm{ij}$ \\
\hline NGBTUN559 & $8.90 \mathrm{fgh}$ & $64.35 \mathrm{ed}$ & 29.00fgh & 23.00fgh & $15.67 \mathrm{ghi}$ & $17.20 \mathrm{fgh}$ & 5.66efg & 28.04fgh & $44.93 \mathrm{efg}$ & 35.93hij & $22.68 \mathrm{jkl}$ & $0.24 \mathrm{fgh}$ & $18.09 \mathrm{klm}$ & $7.20 \mathrm{klm}$ & $3.14 \mathrm{ij}$ \\
\hline NGBTUN560 & 7.00hi & $61.45 \mathrm{ef}$ & $26.20 \mathrm{jkl}$ & $25.20 \mathrm{fgh}$ & $17.15 \mathrm{efg}$ & $19.60 \mathrm{ab}$ & $5.89 \mathrm{ef}$ & 27.63ghi & $34.90 \mathrm{mno}$ & $32.38 \mathrm{klm}$ & 23.98hij & $0.21 \mathrm{hij}$ & 19.39jkl & $6.33 \mathrm{klm}$ & $2.85 \mathrm{j}$ \\
\hline NGBTUN563 & 9.60fgh & $54.37 \mathrm{ghi}$ & $23.10 \mathrm{klm}$ & $24.50 \mathrm{fgh}$ & $14.19 \mathrm{klm}$ & $18,60 \mathrm{abc}$ & $5.28 \mathrm{fgh}$ & 27.39ghi & $41.21 \mathrm{ijk}$ & $35.58 \mathrm{ijk}$ & 28.16def & $0.23 \mathrm{ghi}$ & $19.89 \mathrm{ijk}$ & 7.89ijk & $3.26 \mathrm{ij}$ \\
\hline NGBTUN564 & 7.70fgh & $55.55 \mathrm{ghi}$ & $23.50 \mathrm{klm}$ & 25.60fgh & $14.60 \mathrm{klm}$ & $18.20 \mathrm{bcd}$ & $5.31 \mathrm{fgh}$ & $32.20 \mathrm{a}$ & $39.541 \mathrm{mn}$ & $33.03 \mathrm{jkl}$ & 26.14fgh & $0.19 \mathrm{hij}$ & $15.551 \mathrm{mn}$ & $6.91 \mathrm{klm}$ & $3.34 \mathrm{ij}$ \\
\hline NGBTUN565 & 7.50ghi & 59.80fgh & $25.60 \mathrm{jkl}$ & 25.00fgh & $13.77 \mathrm{klm}$ & $18.40 \mathrm{bcd}$ & 5.36fgh & 29.79abc & $40.82 \mathrm{jkl}$ & $35.22 \mathrm{ijk}$ & $24.37 \mathrm{ghi}$ & $0.31 b c$ & $16.161 \mathrm{mn}$ & $7.40 \mathrm{jkl}$ & 4.70efg \\
\hline NGBTUN566 & $7.70 \mathrm{fgh}$ & 59.80fgh & $21.80 \mathrm{lmn}$ & 23.40fgh & $13.601 \mathrm{mn}$ & 17.40fgh & 4.71hij & 25.10hij & $47.03 \mathrm{def}$ & $40.50 \mathrm{def}$ & $27.35 \mathrm{efg}$ & $0.28 \mathrm{cde}$ & $16.891 \mathrm{mn}$ & 8.82ghi & 4.32fgh \\
\hline NGBTUN567 & 6.70hi & $56.25 \mathrm{ghi}$ & $20.00 \mathrm{mno}$ & 23.40fgh & $11.951 \mathrm{mn}$ & $15.60 \mathrm{fgh}$ & $4.11 \mathrm{ijk}$ & $32.10 \mathrm{a}$ & $39.461 \mathrm{mn}$ & 36.99ghi & $28.05 \mathrm{def}$ & $0.24 \mathrm{fgh}$ & $14.37 \mathrm{mn}$ & $7.70 \mathrm{ijk}$ & 4.98def \\
\hline NGBTUN571 & 7.40hi & $59.10 \mathrm{fgh}$ & $18.00 \mathrm{mno}$ & 24.40fgh & $11.001 \mathrm{mn}$ & 16.40fgh & $3.33 \mathrm{k}$ & $23.15 \mathrm{ijk}$ & $33.02 \mathrm{no}$ & $28.841 \mathrm{mn}$ & $21.02 \mathrm{jkl}$ & $0.17 \mathrm{hij}$ & $18.39 \mathrm{klm}$ & $5.031 \mathrm{~m}$ & $2.87 \mathrm{j}$ \\
\hline NGBTUN572 & $4.00 \mathrm{i}$ & $49.50 \mathrm{jkl}$ & $12.10 \mathrm{pq}$ & 25.00fgh & $9.75 n$ & $17.20 \mathrm{fgh}$ & $3.37 \mathrm{k}$ & $22.40 \mathrm{jk}$ & 29.480 & $26.58 \mathrm{mn}$ & 23.19ijk & $0.54 \mathrm{a}$ & $13.02 \mathrm{n}$ & $7.18 \mathrm{klm}$ & 3.88ghi \\
\hline $\mathrm{CV}(\%)$ & 34.44 & 14.74 & 22.83 & 13.58 & 22.65 & 12.27 & 18.63 & 16.03 & 14.83 & 15.66 & 29.11 & 46.82 & 23.14 & 27.64 & 19.37 \\
\hline$F$ value & 9.47 & 25.30 & 17.98 & 2.72 & 19.65 & 3.52 & 25.01 & 5.94 & 17.70 & 14.14 & 9.03 & 8.73 & 5.97 & 10.34 & 12.62 \\
\hline
\end{tabular}

Relations between quantitative traits were expressed in the correlation matrix (Table 3). According to this table, 36 morphological features were significantly correlated at the 0.05 or 0.001 significance levels. The main positive correlation appeared as follows: length of primary basal leaflet (LPBL) with leaf length (LL; r=0.86) and leaf width (LW; r=0. 77); petiole thickness (PT) with leaf length (LL; $r=0.84)$, leaf width ( $\mathrm{LW} ; \mathrm{r}=0.77$ ) and length of primary basal leaflet (LPBL; $r=0.94)$; root diameter at the shoulder (RDS) with root diameter at the medium (RDMd; $\mathrm{r}=0.94)$ and cortex diameter (RCortD, $\mathrm{r}=0.88$ ); root diameter at the medium (RDMd) with cortex diameter (RCortD, $r=0.89)$. Root weight $(\mathrm{RW})$ was positively and significantly correlated with all root diameters (RDS, RDMd and RDTi) with Pearson coefficients of 0.51 or 0.53 . Positive correlation between root weight and root diameter ( $\mathrm{r}=0.84)$ was also observed in Iranian yellow carrot accessions (Kasiriet al., 2013). Information about the correlation and linkage among different horticultural characteristics is of primary importance in the field of crop improvement. Linkage relationships can be used to increase breeding efficiency by allowing earlier selection and reducing plant population size during selection (Nasrabadiet al., 2012).

\section{Table 3:Pearson correlation coefficients among 15 quantitative traits of 33 Tunisian carrot landraces.}

\begin{tabular}{|c|c|c|c|c|c|c|c|c|c|c|c|c|c|c|}
\hline & $L N$ & $L L$ & $L W$ & $L l N$ & $L P B L$ & $N S P B L$ & $P T$ & $R L$ & $R D S$ & $R D M d$ & $R D T i$ & $R W$ & $R C D$ & RCortD \\
\hline $\mathrm{LL}$ & $-0.41^{*}$ & & & & & & & & & & & & & \\
\hline$\overline{L W}$ & 0.32 & $0.61 * *$ & & & & & & & & & & & & \\
\hline LIN & 0.16 & $0.57 * *$ & $0.67 * *$ & & & & & & & & & & & \\
\hline$\overline{\mathrm{LPBL}}$ & -0.06 & $0.86^{* * *}$ & $0.77 * *$ & $0.69 * *$ & & & & & & & & & & \\
\hline NSPBL & 0.28 & 0.04 & 0.28 & 0.37 & 0.16 & & & & & & & & & \\
\hline PT & -0.12 & $0.84 * *$ & $0.77 * *$ & $0.66^{* *}$ & $0.94 * *$ & 0.07 & & & & & & & & \\
\hline $\mathrm{RL}$ & 0.12 & 0.23 & $0.41 *$ & 0.21 & 0.29 & 0.11 & 0.32 & & & & & & & \\
\hline RDS & 0.32 & -0.08 & 0.15 & 0.01 & 0.01 & $0.44 *$ & -0.06 & -0.21 & & & & & & \\
\hline RDMd & 0.19 & 0.01 & 0.20 & 0.02 & 0.08 & $0.43^{*}$ & -0.00 & -0.21 & $0.94 * *$ & & & & & \\
\hline RDTi & -0.01 & -0.2 & $\begin{array}{l}-0.12 \\
\end{array}$ & $\begin{array}{l}-0.10 \\
\end{array}$ & $\begin{array}{l}-0.27 \\
\end{array}$ & $0.39^{*}$ & $\begin{array}{l}-0.34 \\
\end{array}$ & -0.26 & $0.68^{*}$ & $0.71 * *$ & & & & \\
\hline RW & 0.04 & 0.08 & 0.18 & 0.22 & 0.08 & $0.51^{*}$ & 0.01 & 0.01 & $0.51^{*}$ & $0.53 * *$ & $0.51^{*}$ & & & \\
\hline $\mathrm{RCD}$ & 0.34 & 0.19 & 0.45 & 0.26 & $0.41^{*}$ & $0.38^{*}$ & 0.30 & -0.08 & $0.61 * *$ & $0.65^{* *}$ & 0.19 & 0.32 & & \\
\hline RCortD & 0.34 & -0.24 & 0.02 & -0.04 & -0.14 & $0.47^{*}$ & -0.20 & -0.24 & $0.88 * *$ & $0.89 * * *$ & $0.61^{*}$ & $0.61 * *$ & $0.62^{* *}$ & \\
\hline $\mathrm{RF}$ & 0.26 & $\begin{array}{c}-0.04 \\
\end{array}$ & 0.10 & 0.00 & 0.10 & $\begin{array}{l}-0.16 \\
\end{array}$ & 0.01 & \begin{tabular}{|c|}
-0.17 \\
\end{tabular} & $0.36^{*}$ & $0.39^{*}$ & 0.15 & 0.06 & $0.37^{*}$ & $0.34 *$ \\
\hline
\end{tabular}

\footnotetext{
** Significant at $0.1 \%,{ }^{*}$ significant at $5 \%$.
} 
Because the quantitative characters are interrelated, we conducted a principal component analysis to determine their impact. The first three principal components accounted for $71.71 \%$ of the variance (Table 4). The first principal component with an eigenvalue of 4.88 explained $32.57 \%$ of the total variability and was mainly associated with root diameter at shoulder (RDS), root diameter at the medium (RDMd) and core diameter (RCD). Principal component 2 with an eigenvalue of 4.39 accounted for $29.28 \%$ of the morphological variability and was strongly correlated with petiole thickness (PT), length of primary basal leaflet (LPBL) and leaf length (LL). Principal component 3 with an eigenvalue of 1.47 accounted for $9.85 \%$ of the total variability and was positively correlated with leaf number (LN) and root firmness (RF) but negatively correlated with root diameter at the tip (RDTi) and root weight (RW). The PCA scatterplot defined by the two principal components 1 and 2 (Figure2) separated carrot landraces into 3 groups. The first group (G1) included accessions NGBTUN512, 514, 520, 521, 522, 523, 524 and 525from Monastir (Moknine and Teboulba locations). The second group G2 is formed by the remaining accessions expect for accession NGBTUN540 from Sfax (Hezag) which diverges from all the other accessions and formed the group G3. This accession consistently showed highest values for six quantitative traits (Table 2). The principal component analysis permitted the subdivision of the accessions independently from their geographic zones and their bioclimatic conditions. The quantitative traits may be modified variously by the environmental conditions and are usually governed by many factors or genes each contributing such a small amount of phenotype such that their individual effects cannot be detected by Mendelian methods. They do not show clear differences between individuals and form a spectrum of phenotypes which blend imperceptivity from one type to another as continuous variation (Hill, 2010).

Table 4: Values of the first three components of PCA based on morphological quantitative characters of Tunisian carrot landraces.

\begin{tabular}{|l|c|c|c|}
\hline Principal component & Axis 1 & Axis 2 & Axis 3 \\
\hline Eigenvalue & 4.88 & 4.39 & 1.47 \\
\hline Percentage (\%) & 32.57 & 29.28 & 9.85 \\
\hline Cumulative percentage & 32.57 & 61.85 & 71.71 \\
\hline Character & \multicolumn{2}{|c|}{ Eigenvalue } \\
\hline LN & 0.16 & -0.44 & 0.65 \\
\hline LL & 0.05 & 0.40 & -0.29 \\
\hline LW & 0.19 & 0.37 & 0.17 \\
\hline LIN & 0.14 & 0.34 & -0.01 \\
\hline LPBL & 0.12 & 0.43 & -0.02 \\
\hline NSPBL & 0.28 & 0.02 & -0.15 \\
\hline PT & 0.07 & 0.44 & -0.04 \\
\hline
\end{tabular}


International Journal of Agriculture, Environment and Bioresearch

Vol. 4, No. 04; 2019

ISSN: $2456-8643$

\begin{tabular}{|l|c|c|c|}
\hline RL & -0.04 & 0.22 & 0.12 \\
\hline RDS & 0.40 & -0.13 & -0.01 \\
\hline RDMd & 0.41 & -0.10 & -0.08 \\
\hline RDTi & 0.27 & 0.23 & -0.31 \\
\hline RW & 0.30 & -0.03 & -0.31 \\
\hline RCD & 0.35 & 0.08 & 0.19 \\
\hline RCortD & 0.33 & -0.19 & 0.01 \\
\hline RF & 0.17 & -0.43 & 0.39 \\
\hline
\end{tabular}

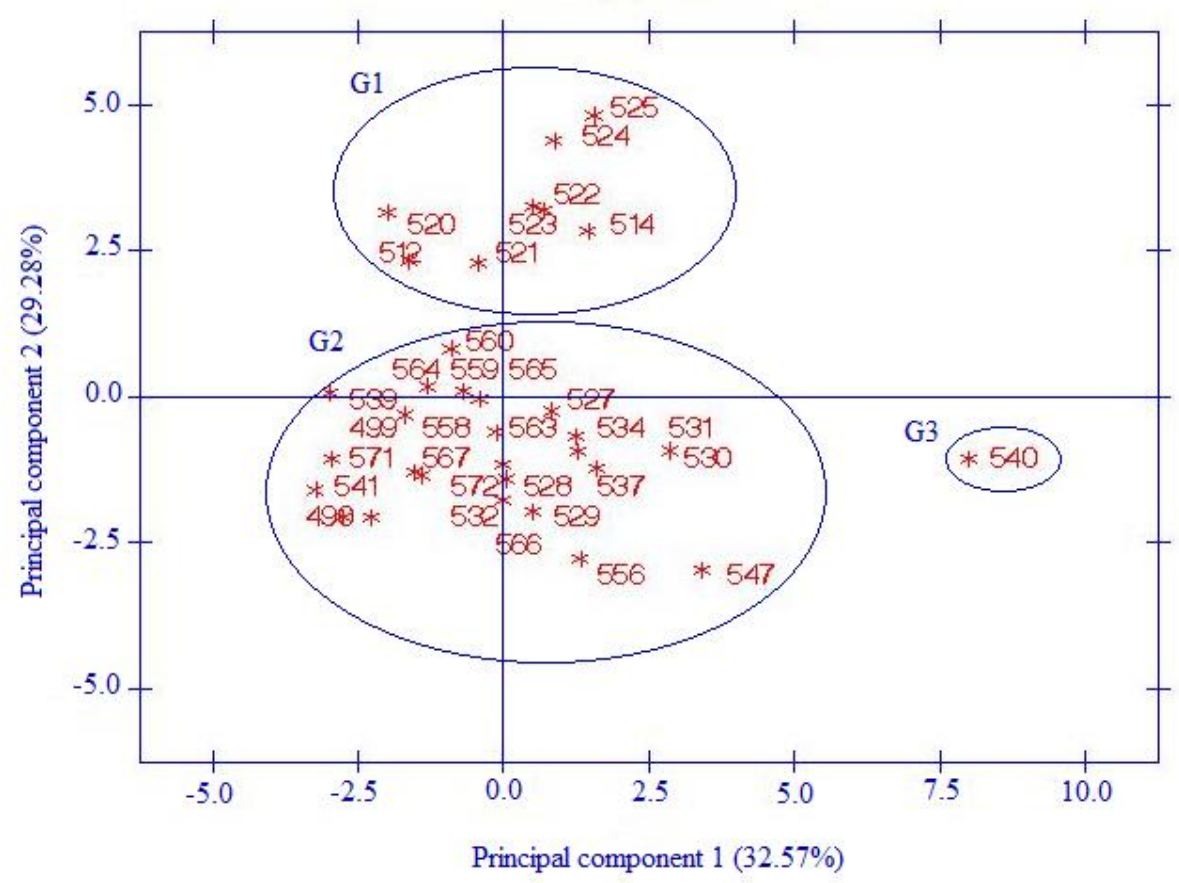

Figure 2:Scatter plot grouping of 33 Tunisian carrot landraces based on the first two principal components of PCA.

\subsubsection{Qualitative characters}

A factorial analysis of correspondence (FAC) was carried out to detect associations and oppositions existing between carrot landraces and qualitative traits, measuring their contribution to the total variability for each factor. Table 5 shows the eigenvalue and cumulative percentage 
of qualitative traits of the first three factors. Factor 1 accounted $22.59 \%$ of the total variance and was positively correlated with root external colour (REC), extent of green colour of shoulder skin (REGCSS), root branching (RB) and anthocyanin colouration of shoulder skin (RACSS). Factor 2 explained $17.52 \%$ of the total variance and was positively correlated with foliage coverage (FC), root shoulder shape (RSS), protrusion above soil (RPAS) and core colour compared to root colour (RCCCC). The scatter plot of factorial correspondence analysis defined by the first two factors (Figure3) divided carrot accessions on the basis of the qualitative characteristics into four distinct groups. The first group (G1) included accessions NGBTUN547 and 556 from Gabes, NGBTUN564, 565, 566, 567 from Nabeul (Slimane location); NGBTUN571 and 572 from Siliana characterized as having a narrow crown width; leaves with a strong anthocyanin petiole colouration, a fine division and a medium intensity of green colour; and roots with orange skin and core colour, a small extent of green colour of shoulder skin havinga rounded shape. Accessions from Gabes are characterized by a blunt root tip and a medium obtriangular root shape in longitudinal section. Whereas accessions from Siliana and Slimane exhibited a slightly pointed root tip and a narrow oblong root shape in longitudinal section. The second group (G2) formed by accessions NGBTUN558, 559, 560, 563 from Nabeul (Menzel Temime) and NGBTUN490, 499 from Nabeul (Korba) presented leaves with coarse division and medium hairiness but without anthocyanin petiole colouration. Roots are bent and havea yellow external colour, a very weak surface ridging, and a large extent of green colour of shoulder skinwhich is characterized by a conical shape. The third group (G3) comprised accessions NGBTUN527, 528, 529, 530, 531 from Sidi Bouzid, NGBTUN532, 534, 537, 539 from Kairouan and NGBTUN540, 541 from Sfax presented leaves with a medium division, a strongly hairiness, and a slightly to intermediate coloured petiole. Roots are pinkish red in external colour with a narrow obtriangular to narrow oblong shape in longitudinal section, a weak surface ridging, a flat shoulder shape with a very small extent of skin green colour. The fourth group (G4) formed by NGBTUN512, 514, 520, 521, 522, 523, 524 and 525 from Monastir showed an intermediate to a wide foliage width and intensely dark green leaves. Roots are yellow to orange with a strongly pointed tip shape, a white to yellow core colour, a large extentof green colourof shoulder skin and a sparse to intermediate branching. Among these accessions, there are roots with purplecolourexternally. Accessions in the first group are assembled independently of their geographic origin; this could be explained by the allogamous mating system of the species or the frequent seed exchange among farmers and regional markets (Mezghaniet al., 2014). However, accessions of the second, the third and the firth group are from the same geographic zone, this could be explain by a local human selection or a suitable adaptation of accessions to their specific habitat conditions. 
International Journal of Agriculture, Environment and Bioresearch

Vol. 4, No. 04; 2019

ISSN: $2456-8643$

Table 5: Values of the first three factors of FCA based on morphological qualitative characters of Tunisian carrot landraces.

\begin{tabular}{|c|c|c|c|}
\hline Principal factor & Factor 1 & Factor 2 & Factor 3 \\
\hline Eigenvalue & 0.44 & 0.38 & 0.31 \\
\hline Percentage (\%) & 22.59 & 17.52 & 11.29 \\
\hline Cumulative percent & 22.59 & 40.12 & 51.4 \\
\hline Character & \multicolumn{2}{|c|}{ Eigenvalue } & \\
\hline RA & 0.56 & 0.37 & \\
\hline RSLS & 0.32 & -0.11 & \\
\hline RSS & 0.64 & 1.10 & \\
\hline RTS & 0.28 & -0.22 & \\
\hline REC & 1.18 & 0.50 & \\
\hline $\mathrm{RB}$ & 1.10 & -0.70 & \\
\hline RACSS & 1.10 & -0.01 & \\
\hline REGCSS & 1.12 & 0.04 & \\
\hline RSR & 0.33 & 0.16 & \\
\hline RCC & 0.59 & -0.21 & \\
\hline RCCCC & -0.46 & 1.09 & \\
\hline RFCDTS & 0.10 & 0.01 & \\
\hline $\mathrm{FC}$ & -0.01 & 1.12 & \\
\hline RPAS & 0.21 & 1.09 & \\
\hline $\mathrm{CW}$ & 0.40 & -0.55 & \\
\hline LD & 0.16 & 0.05 & \\
\hline LIGC & 0.69 & -0.39 & \\
\hline PCP & 0.34 & 0.13 & \\
\hline LH & 0.20 & 0.18 & \\
\hline FW & -0.21 & 0.30 & \\
\hline
\end{tabular}




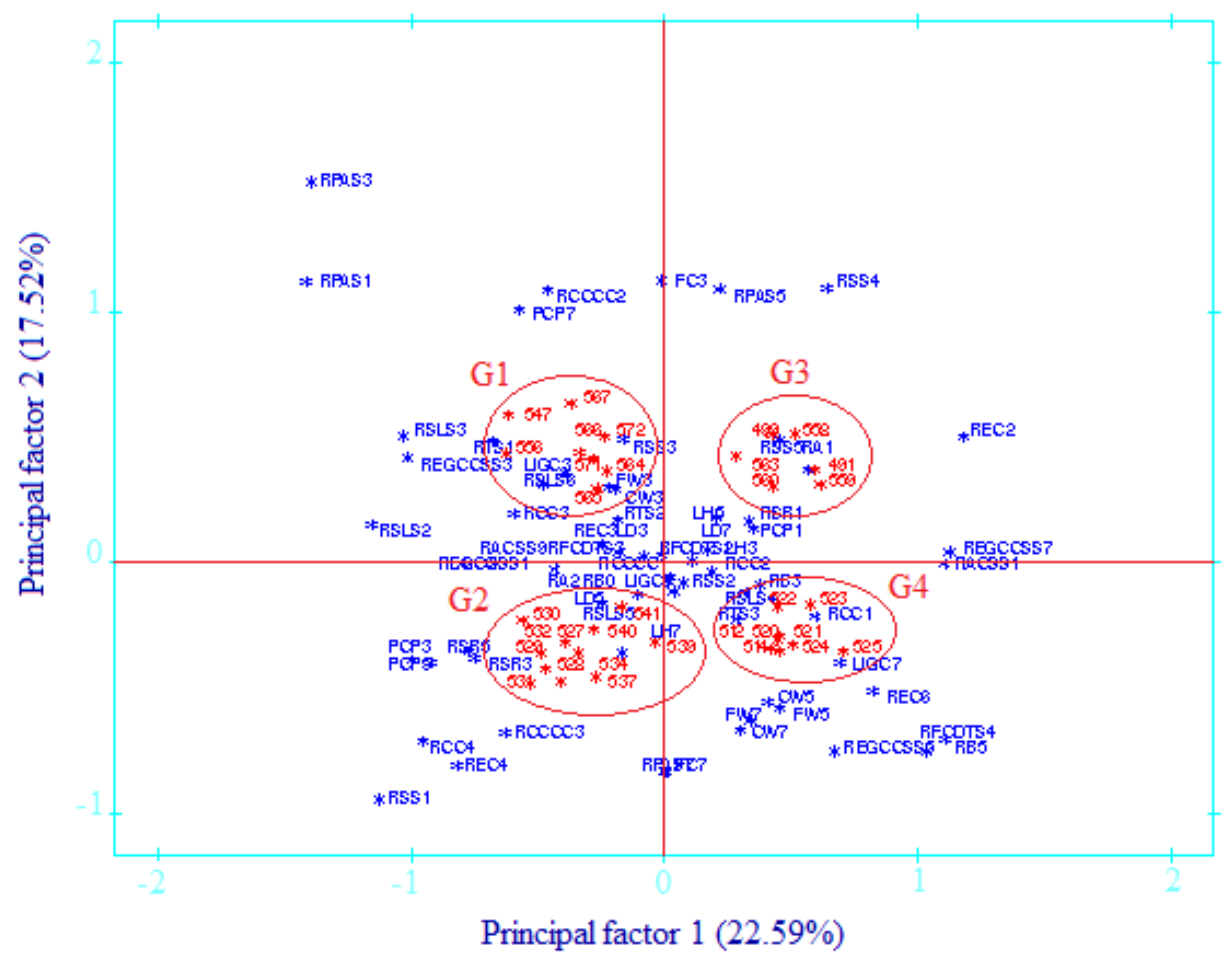

Figure 3: Scatter plot grouping of 33 Tunisian carrot landraces based on the first two principal factors of FCA.

\subsection{Grouping of landraces using quantitative and qualitative characters}

A dendrogram (Figure4) combining quantitative and qualitative characters was carried out to evaluate the general pattern of variance and to establish relationship among carrot landraces. At an average distance of 1.0, hierarchical clustering defines two major clusters including the same groups (G1 to G4) identified by FCA. Landraces of G2 from Nabeul (Menzel Temime and Korba) and G4 from Monastir (Moknineand Teboulba) fell together in cluster Cl1 whereas cluster Cl2 included landraces from Sidi Bouzid, Kairouan, Sfax (G3) and Gabes, Slimane (Nabeul) andSiliana (G1). This hierarchical classification provided evidence that landraces are clustered independently to their geographic origins. Abdellaouiet al. (2010) and Lahbibet al. (2013) reported that the cluster pattern of barley and pepper landraces in Tunisia is not always related to geographical distribution. Diversity detected within accessions could mainly be attributed to diverse agro-climatic conditions in Tunisia. The intraregional and interregional diversity may be as a valuable source for crop improvement (Lahbibet al., 2012). 
Vol. 4, No. 04; 2019

ISSN: $2456-8643$

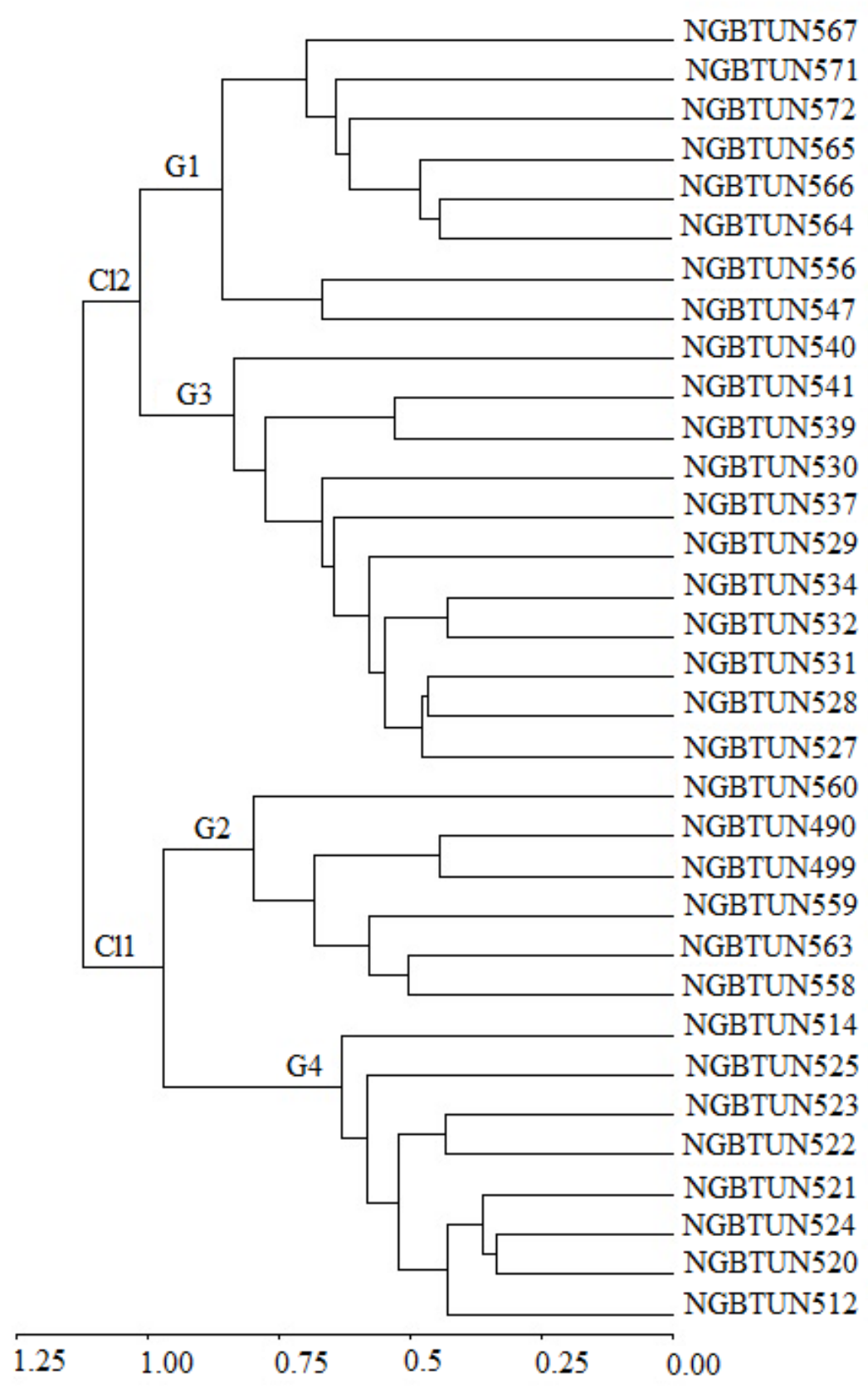

Average distance between clusters

Figure 4: Dendrogram obtained from cluster analysis of 33 Tunisian carrot landraces using the UPGMA. 


\section{CONCLUSION}

The present study uses morphological characterization of 33 Tunisian carrot landraces collected from diverse regions of Tunisia to evaluate quantitative and qualitative parameters related to roots and leaves. We here document a high morphological variability within landraces. These results, in combination with previous ones (Mezghaniet al., 2014, 2017) confirm that Tunisia is a principal major centerof diversification for Daucus in the Mediterranean region. This information will be helpful to curators in the management and improvement of carrot germplasm in Tunisia and worldwide.

\section{Acknowledgment}

The authors are grateful to all engineers and technicians from the 'Commissariat Regional de Développement Agricole' and the 'Cellule Territoriale de vulgarisation Agricole' in the visited regions for their contribution in the survey and collection missions.

\section{REFERENCES}

[1] Abdellaoui, R., Kadri, K., Ben Naceur, M., Bettaib Ben Kaab, L. (2010). Genetic diversity in some Tunisian barley landraces based on RAPD markers. Pakistan Journal of Botany 42(6):3775-3782.

[2] Al Khanjari, S., Filatenko, A.A., Hammer, K., Buerkert, A. (2008). Morphological spike diversity of Omani wheat. Genetic Resources and Crop Evolution 55:1185-1195.

[3] Banga, O. (1957). Origin of the European cultivated carrot. Euphytica 6:54-63.

[4] Banga, O. (1963). Origin and domestication of the western cultivated carrot. GeneticaAgraria 17:357-370.

[5] DGPA. (2015). Annual report of General Directorate for Agricultural Production, the Ministry of Agriculture, 2015.

[6] Eticha, F., Bekele, E., Belay, G., Borner, A. (2005). Phenotypic diversity in durum wheat collected from Bale and Wello regions of Ethiopia. Plant Genetic Resources 3(1):35-43.

[7] Hamrick, J.L., Linhart, Y.B., Mitton, J.B. (1979). Relationships between life history characteristics and electrophoretically detectable genetic variation in plants. Annual Review of Ecology and Systematics 10:173-200.

[8] Hill, W.G. (2010). Understanding and using quantitative genetic variation. Philosophical Transactions of the Royal Society ofLondondon. Series B, Biological sciences 365(1537):73-85. 
[9] Iorizzo, M., Senalik, D.A., Ellison, S.L., Grzebelus, D., Cavagnaro, P.F., Allender, C., Brunet, J., Spooner, D.M., Van Deynze, A., Simon, P.W. (2013). Genetic structure and domestication of carrot (Daucus carota subsp. sativus L.) (Apiaceae). American Journal of Botany 100:930-938.

[10] IPGRI. (1998). Descriptors for wild and cultivated carrot (Daucus carota L.). International Plant Genetic Resources Institute, Rome.

[11] Jaradat, A.A., Shahid, M., Al Maskri, A.Y. (2004). Genetic diversity in the Batini barley landrace from Oman: I. Spike and seed quantitative and qualitative traits. Crop Science 44:304315 .

[12] Kasiri, M.R., Hassandokht, M.R., Kashi, A., Shahi Gharahlar, A. (2013). Evaluation of genetic diversity in Iranian yellow carrot accessions (Daucus carota var. sativus), an exposed to extinction rooty vegetable, using morphological characters. International Journalof Agricultre and Crop Science 6(3):151-156.

[13] Lahbib, K., Bnejdi, F., El Gazzah, M. (2012). Genetic diversity evaluation of pepper (Capsicum annuum L.) in Tunisia based on morphologic characters. African Journalof Agricultural Research 23:3413-3417.

[14] Lahbib, K., Bnejdi, F., El Gazzah, M. (2013). Selection of pepper parent from a collection of Capsicum annuum landraces based on genetic diversity. Journalof Plant Breeding and Crop Science 5(5):68-72.

[15] Le Floc'h, E., Boulos, L., Vela, E. (2010). Catalogue synonymique commente' de la flore de Tunisie. Banque Nationale des Ge`nes de la Tunisie, Tunis.

[16] Maksylewicz, A., Baranski, R. (2013). Intra-population genetic diversity of cultivated carrot (Daucus carota L.) assessed by analysis of microsatellite markers. Acta Biochimica Polonica 60(4):753-60.

[17] Mehrabi, Z., Hassandokht, M.R., Khosrwchahli, M. (2014). Assessment of genetic diversity based on morphological characters in some Iranian yellow carrot accessions (Daucus carota L. sativus). International Journal of Biosciences 5(5):33-42.

[18] Mengistu, D.K., Kiros, A.Y., Pe', M.E. (2015) .Phenotypic diversity in Ethiopian durum wheat (Triticum turgidum var. durum) landraces. The Crop Journal 3:190-199.

[19] Mezghani, N., Zaouali, I., Bel Amri, W., Rouz, S., Simon, P.W., Hannachi, C.H., Gharbi, Z., Neffati, M., Bouzbida, B., Spooner, D.M. (2014). Fruit morphological descriptors as a tool for discrimination of Daucus L. germplasm. GeneticResources and Crop Evolution 61:499-510. 
[20] Mezghani, N., Ben Amor, J., Spooner, D.M., Boubaker, H., Najmi, A.M., Rouz, S., Hannachi, C.H., Neffati, M., Tarchoun, N. (2017). Multivariate analysis of morphological diversity among closely related Daucus species and subspecies in Tunisia. Genetic Resources and Crop Evolution 64:2145-2159.

[21] Nasrabadi, H.N., Nemati, H., Sobhani, A., Sharifi, M. (2012). Study on morphologic variation of different Iranian melon cultivars (Cucumis melo L.). African Journal of Agricultural Research 7(18):2764-2769.

[22] Peterson, C.E., Simon, P.W. (1986). Carrot Breeding. In: Basset MJ (ed) Breeding vegetable crops. AVI Publishing Company, Westport, pp 321-356.

[23] Pottier Alapetite, G. (1979). Daucus. Flore de la Tunisie, Angiospermes-Dicotyledones, Apetales, Dialypetales. ImprimerieOfficielle de la Re'publiqueTunisienne, Tunis, pp 615-621.

[24] Shannon, C.E., Weaver, W. (1949). The mathematical theory of communication. University of Illinois Press, Urbana.

[25] Simon, P.W. (1990). Carrots and other horticultural crops as a source of provitamin A carotenes. HortScience 25:1495-1499.

[26] Stolarczyk, J., Janick, J. (2011). Carrot: History and iconography. Chron Horticult 51:1318.

[27]n UPOV. (2007). Carotte (Daucus carota L.) Principes directeurs pour la Conduite de l'Examen de la Distinction, de l'Homogénéité et de la Stabilité. International Union for the Protection of New Varieties of Plants.

[28] Vaughan, J.G., Geissler, C.A. (2009). The new Oxford book of food plants. Oxford University Press, Oxford. 